\title{
Anharmonic stabilization and band gap renormalization in the perovskite CsSnl3
}

\author{
Patrick, Christopher; Jacobsen, Karsten Wedel; Thygesen, Kristian Sommer
}

Published in:

Physical Review B

Link to article, DOI:

10.1103/physrevb.92.201205

Publication date:

2015

Document Version

Publisher's PDF, also known as Version of record

Link back to DTU Orbit

Citation (APA):

Patrick, C., Jacobsen, K. W., \& Thygesen, K. S. (2015). Anharmonic stabilization and band gap renormalization in the perovskite CsSnl 3 . Physical Review B, 92(20), [201205]. https://doi.org/10.1103/physrevb.92.201205

\section{General rights}

Copyright and moral rights for the publications made accessible in the public portal are retained by the authors and/or other copyright owners and it is a condition of accessing publications that users recognise and abide by the legal requirements associated with these rights.

- Users may download and print one copy of any publication from the public portal for the purpose of private study or research.

- You may not further distribute the material or use it for any profit-making activity or commercial gain

- You may freely distribute the URL identifying the publication in the public portal

If you believe that this document breaches copyright please contact us providing details, and we will remove access to the work immediately and investigate your claim. 


\title{
Anharmonic stabilization and band gap renormalization in the perovskite $\mathrm{CsSnI}_{3}$
}

\author{
Christopher E. Patrick, Karsten W. Jacobsen, and Kristian S. Thygesen \\ Center for Atomic-Scale Materials Design (CAMD), Department of Physics, Technical University of Denmark, \\ DK-2800 Kongens Lyngby, Denmark
}

(Received 30 July 2015; published 20 November 2015)

\begin{abstract}
Amongst the $X(\mathrm{Sn}, \mathrm{Pb}) Y_{3}$ perovskites currently under scrutiny for their photovoltaic applications, the cubic B- $\alpha$ phase of $\mathrm{CsSnI}_{3}$ is arguably the best characterized experimentally. Yet, according to the standard harmonic theory of phonons, this deceptively simple phase should not exist at all due to rotational instabilities of the $\mathrm{SnI}_{6}$ octahedra. Here, employing self-consistent phonon theory, we show that these soft modes are stabilized at experimental conditions through anharmonic phonon-phonon interactions between the Cs ions and their iodine cages. We further calculate the renormalization of the electronic energies due to vibrations and find an unusual opening of the band gap, estimated as 0.24 and $0.11 \mathrm{eV}$ at 500 and $300 \mathrm{~K}$, which we attribute to the stretching of Sn-I bonds. Our work demonstrates the important role of temperature in accurately describing these materials.
\end{abstract}

DOI: 10.1103/PhysRevB.92.201205

PACS number(s): 63.20.Ry, 64.60.Ej

Four decades after its identification as an unusual phasechange material [1], the inorganic perovskite $\mathrm{CsSnI}_{3}$ has experienced a revival of interest in its technological applications. After being used as a hole transporter in solid-state photovoltaics [2], the subsequent explosion in activity surrounding perovskite solar cells [3] has seen $\mathrm{CsSnI}_{3}$ incorporated into new devices as a lead-free light absorber [4] with favorable optical properties [5-8]. Like many perovskites [9] $\mathrm{CsSnI}_{3}$ has a rich phase diagram, driven by low-energy rotations and tilts of the $\mathrm{SnI}_{6}$ octahedra [10-12]. In addition $\mathrm{CsSnI}_{3}$ has an unusual electronic structure, with a nondegenerate and highly-dispersive valence band [13] and an intra-atomic band gap strongly coupled to external strain [13-15].

In a wider context, $\mathrm{CsSnI}_{3}$ is the gateway to understanding the basic physics of the family of $X(\mathrm{Sn}, \mathrm{Pb}) Y_{3}$ perovskites ( $X=$ cation, $Y=$ halogen). Unlike its famous cousin $\mathrm{MAPbI}_{3}$ $\left(\mathrm{MA}=\right.$ methylammonium), $\mathrm{CsSnI}_{3}$ has (i) no permanent cationic dipole moment [16], (ii) reduced spin-orbit coupling due to the lighter mass of Sn [17], and (iii) a high-symmetry cubic $(\mathrm{B}-\alpha)$ phase characterized by many studies $[10,12,18]$. However, theoretical investigations [11,19-21] consistently find the B- $\alpha$ phase to be unstable against spontaneous rotation of the $\mathrm{SnI}_{6}$ octahedra, so on energetic grounds this phase should not exist at all. The answer to this puzzle must partly lie in the fact that the B- $\alpha$ phase is stable only at high temperature [12], where both energetic and entropic contributions determine the free energy $F$. Unfortunately the most widely-used approach of calculating $F$ from first principles, the quasiharmonic approximation [22], cannot be straightforwardly applied [21] due to the presence of the unstable (imaginary) phonon modes (Fig. 1).

In this Rapid Communication, we demonstrate the critical role played by anharmonicity in stabilizing the experimentallyobserved cubic and tetragonal (B- $\alpha$ and B- $\beta$ ) phases of $\mathrm{CsSnI}_{3}$. We perform our $a b$ initio investigation using a stochastic implementation of self-consistent phonon theory [24-27]. We show that the $\mathrm{SnI}_{6}$ octahedra are stabilized against tilts and rotations by interacting with the renormalized vibrations of the Cs ions. Unexpectedly our calculations also reveal a temperature-induced opening of the band gap, with a magnitude of 0.24 and $0.11 \mathrm{eV}$ at 500 and $300 \mathrm{~K}$, respectively. The significant size of these corrections (36 and $11 \%$ of the uncorrected gaps) places temperature effects at a similar level of importance as spin-orbit coupling for determining the band gap in these materials [17], yet usually they are not included in ab initio studies. We further find that the gap opening is not consistent with a harmonic theory of band gap renormalization [28] but can be understood in terms of an increase in average length of the Sn-I bonds.

All total energy and force calculations in this paper were performed within a generalized-gradient approximation to density-functional theory (the PBEsol functional [29]), expanding the wave functions in a plane-wave basis set [30] and treating the interactions between electrons and ion cores within the projector-augmented wave formalism [31] as implemented in the GPAW code [32].

In Fig. 1 we show the phonon band structure obtained for the B- $\alpha$ phase calculated in the harmonic approximation using the finite displacement method [33,34]. Instabilities corresponding to tilts and rotations of the $\mathrm{SnI}_{6}$ octahedra are found at the $M$ and $R$ points of the Brillouin zone [11]. One of the triply-degenerate soft modes at $M$ is shown in Fig. 2, together with a frozen-phonon calculation of the potential energy surface (PES) with respect to a distortion $x_{\text {rot }}$ along this mode. The cubic structure $\left(x_{\text {rot }}=0\right)$ is metastable, and the system can lower its potential energy through an octahedral rotation to a new structure with tetragonal symmetry. These soft modes have been observed under a number of different computational setups and theoretical approximations [11,19-21] and cannot be stabilized for example by the application of a strain. In fact increasing the lattice constant above its $0 \mathrm{~K}$ equilibrium value yields further soft modes, identified as ferroelectric instabilities in Ref. [21].

The fact that experiments observe only the cubic phase at temperatures above $440 \mathrm{~K}[10,12,18]$ indicates that this phase corresponds to a minimum of the free energy $F$. In the quasiharmonic approximation [22], $F$ is replaced with $\tilde{F}(\omega, T)$, the free energy of an ensemble of oscillators of temperature $T$ with frequencies $\boldsymbol{\omega}=\left\{\omega_{1}, \omega_{2}, \ldots \omega_{v}\right\}$ :

$$
\tilde{F}(\boldsymbol{\omega}, T)=V_{0}+\sum_{\nu}\left[\frac{\hbar \omega_{\nu}}{2}-k_{B} T \ln \left[1+n_{B}\left(\omega_{\nu}, T\right)\right] .\right.
$$



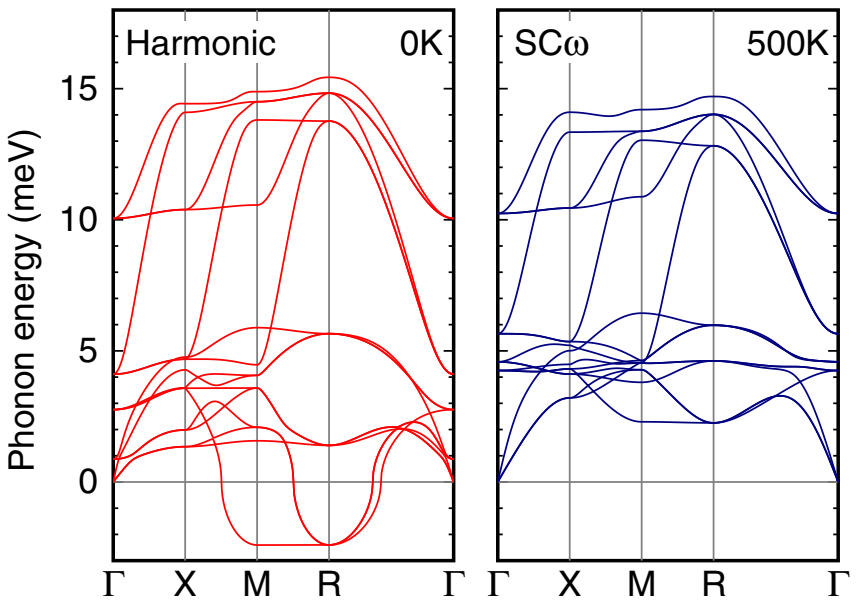

FIG. 1. (Color online) Phonon band structures obtained for the B- $\alpha$ phase of $\mathrm{CsSnI}_{3}$ calculated under the harmonic approximation or with self-consistent phonon frequencies ( $\mathrm{SC} \omega)$. Imaginary frequencies are shown as negative. The supercell calculations do not include the nonanalytic correction accounting for the longwavelength splitting of polar modes [23].

$V_{0}$ is the energy of the ions in their equilibrium positions, $\hbar$ and $k_{B}$ the Planck and Boltzmann constants, and $n_{B}$ the Bose-Einstein distribution function.

A quasiharmonic treatment of the B- $\alpha$ phase would replace $\omega$ with the phonon eigenfrequencies shown in Fig. 1, but there are two difficulties: First, Eq. (1) is defined only for real phonon frequencies, so the contribution to $F$ from the soft modes cannot be included. Second, from the harmonic phonon frequencies and eigenvectors of Fig. 1 we calculate that $\mathrm{Cs}$ atoms would undergo typical oscillations with a root mean-square displacement of $0.8 \AA$ at $500 \mathrm{~K}$, corresponding to over $18 \%$ of the distance to their iodine neighbors at equilibrium [35]. Such large displacements are unlikely to be well described within the harmonic approximation.

Determining $F$ for the B- $\alpha$ phase therefore requires moving beyond the (quasi)harmonic approximation. Different approaches to this problem have been developed, including
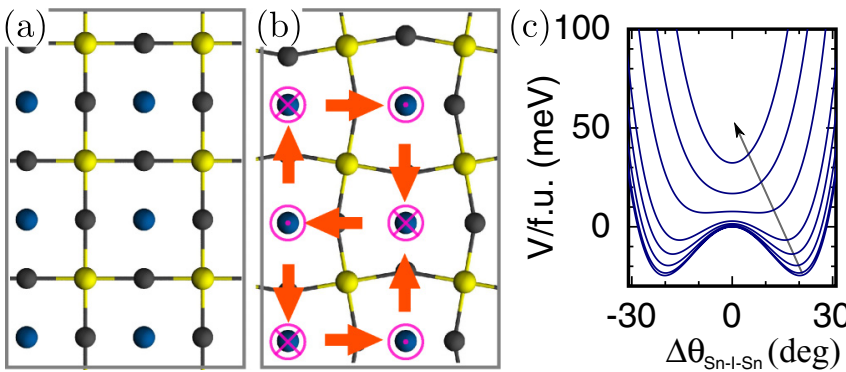

FIG. 2. (Color online) (a) B- $\alpha$ phase of $\mathrm{CsSnI}_{3}$, with blue/yellow/gray atoms $=\mathrm{Cs} / \mathrm{Sn} / \mathrm{I}$. (b) B- $\alpha$ structure distorted along modes at the $M$ point corresponding to $\mathrm{SnI}_{6}$ octahedral rotation (orange arrows) and Cs vibration (pink arrows in/out of page). The respective amplitudes along each mode are $x_{\text {rot }}$ and $x_{\mathrm{Cs}}$. (c) Energy $V$ vs deviation in Sn-I-Sn bond angle $\theta$ for constant values of $x_{\mathrm{Cs}}$ of (in direction of arrow) 0.0, 0.25, 0.5, 0.75, 1.0, 1.5, 2.0 , and 2.5, in units of ${\sqrt{\left\langle x_{\mathrm{CS}}^{2}\right\rangle}}_{T=500 \mathrm{~K}}$. methods based on parametrizations and perturbative expansions of the PES [36-38], molecular dynamics [39,40], and self-consistent phonons [24-27,41]. Here we follow the selfconsistent phonon approach and calculate a fictitious free energy $\mathcal{F}(\omega, T)$ as

$$
\mathcal{F}(\omega, T)=\tilde{F}(\omega, T)+\langle V\rangle_{T}-\langle\tilde{V}\rangle_{T} .
$$

Here $\tilde{V}$ is a harmonic approximation to the true PES $V$, and $\langle A\rangle_{T}$ is a thermal average of a quantity $A$ with respect to the fictitious harmonic system, whose exact value is obtained via Mehler's formula [42,43] as

$$
\langle A\rangle_{T}=\prod_{\nu} \frac{1}{\sqrt{2 \pi\left\langle x_{\nu}^{2}\right\rangle_{T}}} \int d x_{v} e^{-x_{v}^{2} / 2\left\langle x_{v}^{2}\right\rangle_{T}} A(\mathbf{x}) .
$$

$\mathbf{x}$ gives the amplitudes along each phonon mode $v$, with the mean-square amplitude at temperature $T$ given as $\left\langle x_{v}^{2}\right\rangle_{T} . \mathcal{F}$ is the free energy of the real system evaluated on the thermal equilibrium state of the fictitious system and is a rigorous upper bound to the true free energy $F$ [27]. The self-consistent set of frequencies $\boldsymbol{\omega}$ are chosen as those which minimize $\mathcal{F}$.

A fully self-consistent phonon theory (e.g., Refs. [26,27]) also minimizes $\mathcal{F}$ with respect to phonon eigenvectors and equilibrium ionic positions, but in the current study we keep these quantities fixed at their harmonic values. The reasons for performing this simplification are (i) for the high-symmetry B- $\alpha$ phase, many of the phonon eigenvectors (including the soft modes) are fixed by the crystal symmetry, and (ii) the large unit cells and low symmetry of the B- $\beta$ and $\mathrm{B}-\gamma$ phases render a full minimization of $\mathcal{F}$ impractical [44], even after performing the symmetrization techniques of Ref. [27]. Then, as has been done previously for calculating free energies [26,27], absorption spectra [45], and magnetic spectroscopies [46], we evaluate the thermal averages of Eq. (3) stochastically from an ensemble of configurations with ionic displacements distributed according to $\prod_{v} \exp \left[-x_{v}^{2} /\left(2\left\langle x_{v}^{2}\right\rangle_{T}\right)\right]$. We label the current scheme SC $\omega$.

Figure 1 shows the $\mathrm{SC} \omega$-calculated phonon band structure obtained at $500 \mathrm{~K}$. There are three points to note. First, the soft modes at the $M$ and $R$ points are stabilized to positive energies of $2.3 \mathrm{meV}$. Second, the vibrational energies of the $\mathrm{Cs}$ atoms appearing at $1-3 \mathrm{meV}$ in the harmonic approximation [21] are renormalized by more than a factor of two in $\mathrm{SC} \omega$. As a result, ferroelectric instabilities involving $\mathrm{Cs}$ atoms that appear at a strained lattice vanish at high temperatures [35]. Finally, the lattice constant which minimizes $\mathcal{F}$ is calculated to be $6.21 \AA$, which compared to experiment (6.206 $\AA$ [12]) is a significant improvement over the values of $6.131 \AA$ found by minimizing the total energy and $6.160 \AA$ obtained at $500 \mathrm{~K}$ from a quasiharmonic analysis ignoring the soft modes [21].

The significant renormalization of the $\mathrm{Cs}$ vibrations points to the mechanism by which the soft modes are stabilized in $\mathrm{SC} \omega$. The SC $\omega$ potential calculated for octahedral rotations is far steeper than that expected from a one-dimensional analysis of a quartic potential, which yields a parabola wide enough for the system to sample the two minima [35]. Instead one must consider phonon-phonon interactions between the octahedral rotations and the vibrations of the Cs atoms. In Fig. 2(c) we show the PES obtained by simultaneously displacing the $\mathrm{Cs}$ atoms along an $M$-point phonon while rotating the $\mathrm{SnI}_{6}$ octahedra. Harmonically for each Cs mode amplitude 
$x_{\mathrm{Cs}}$ one would expect an identical PES, offset by an energy $1 / 2 M_{P} \omega_{\mathrm{Cs}}^{2} x_{\mathrm{Cs}}^{2}\left(M_{P}\right.$ is the proton mass). Instead, the PES changes shape, showing that terms like $x_{\mathrm{Cs}}^{2} x_{\text {rot }}^{2}$ stabilize the cubic structure. We stress that the analysis of Fig. 2(c) only couples two phonon modes, whilst SC $\omega$ includes all couplings.

Crucially, the value of $\mathcal{F}$ calculated for the cubic phase is $20 \mathrm{meV}$ per formula unit lower than the tetragonally-distorted phase at $500 \mathrm{~K}$, showing that at high temperature it is more beneficial to the free energy to have the Cs atoms vibrating in a large volume than it is to reduce $V_{0}$ by rotating the $\mathrm{SnI}_{6}$ octahedra. Our calculations corroborate the experimental interpretation of Cs atoms "rattling" within the perovskite cages [12].

Given the interest in the optoelectronic properties of $\mathrm{CsSnI}_{3}$, it is desirable to quantify the effects of phonons on the electronic band gap $E_{g}$. There is increasing evidence that semilocal exchange-correlation functionals find a weaker electron-phonon coupling strength compared to more sophisticated theories of electronic excitations, e.g., the $G W$ approximation $[47,48]$. For this reason we perform electronic structure calculations using the derivative discontinuitycorrected GLLB-SC functional of Ref. [49], which has been found to improve the PBEsol description of the band gap for a range of materials $[14,50]$. We calculate a gap deformation potential of $7.20 \mathrm{eV}$ with GLLB-SC, close to the value of $7.35 \mathrm{eV}$ found from the quasiparticle self-consistent $G W$ (QSGW) calculations of Ref. [13] and steeper than the values of 4.73 found with PBEsol or $4.65 \mathrm{eV}$ from the local-density approximation [13]. The derivative discontinuity is responsible for this difference [35].

Expanding the lattice constant from $6.131 \AA$ (harmonic, $T=0 \mathrm{~K})$ to $6.21 \AA$ (SC $\omega, T=500 \mathrm{~K})$ already accounts for an increase of the gap $E_{g}$ from $0.40 \mathrm{eV}$ to $0.66 \mathrm{eV}$. However in addition there is a constant-volume renormalization of the gap due to phonons [51], which in the adiabatic approximation of Ref. [28] is calculated as $\Delta E_{g}=\left\langle E_{g}\right\rangle_{T}-E_{g}^{0}$, where $E_{g}^{0}$ is the gap calculated with the ions in their equilibrium positions [43]. We use Eq. (3) to evaluate $\left\langle E_{g}\right\rangle_{T}$ from the $\mathrm{SC} \omega$ frequencies at the experimental volume at $500 \mathrm{~K}$. The band gaps calculated for 300 configurations is shown in Fig. 3. The calculated $\Delta E_{g}$ is remarkable for being both large and positive, i.e., the electron-phonon interaction increases the gap. Although the latter behavior has been observed experimentally for materials like copper halides [51,52], ab initio calculations of electron-phonon renormalization have so far focused on semiconductors like diamond and $\mathrm{Si}$ where the gap is reduced by temperature [47,53-55].

We have also studied the technologically-relevant low temperature $\mathrm{B}-\beta$ and $\mathrm{B}-\gamma$ phases at 380 and $300 \mathrm{~K}$, respectively. Owing to the close agreement of the SC $\omega$ B- $\alpha$ lattice constant with experiment, we used the experimental lattice constants reported in Ref. [12] for the other phases. We show the SC $\omega$ band structures in the supplemental information [35]. For the $\mathrm{B}-\beta$ phase, the $\mathrm{SC} \omega$ calculations remove the unstable modes and renormalize the frequencies of the Cs modes, whilst for the B- $\gamma$ phase some small changes in phonon frequencies occur across the spectrum [35]. The calculated corrections to the band gap are again large, yielding $+0.70 \mathrm{eV}(\mathrm{B}-\alpha$ phase, $500 \mathrm{~K}),+0.45 \mathrm{eV}(\mathrm{B}-\beta$ phase, $380 \mathrm{~K})$, and $+0.31 \mathrm{eV}(\mathrm{B}-\gamma$ phase, $300 \mathrm{~K})$. However as discussed below these values are

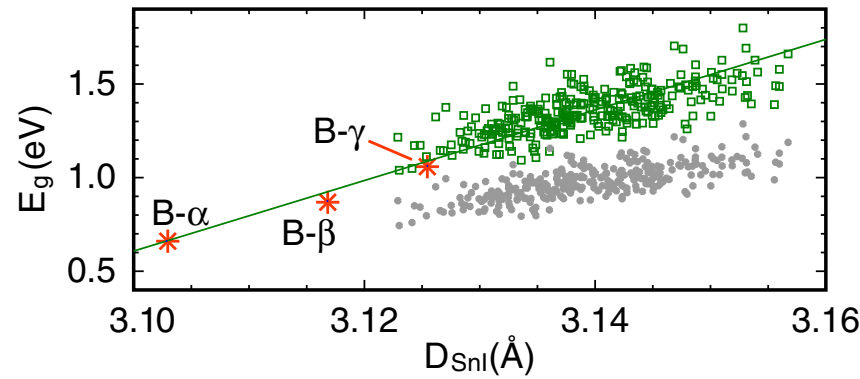

FIG. 3. (Color online) GLLB-SC band gaps calculated with/without (green squares/gray circles) derivative discontinuity contribution for an ensemble of 300 configurations vs average Sn-I bond length $D_{\text {SnI }}$. A linear fit to the data is shown. $E_{g}$ is taken as the difference between the highest occupied state and the average of the three lowest unoccupied states at $R$ [35]. We also show the gaps of the unperturbed B- $\alpha, \mathrm{B}-\beta$, and B- $\gamma$ structures (orange stars). Note that these calculations were performed in a $2 \times 2 \times 2$ supercell and are subject to the finite size effects discussed in the text.

likely to be overestimates due to finite size effects in our supercell calculations.

To further investigate this band gap renormalization we first consider the harmonic theory of Ref. [28], where

$$
\Delta E_{g} \approx \Delta E_{g}^{(2)}=\sum_{v} \frac{\partial E_{g}}{\partial n_{v}}\left[n_{B}\left(\omega_{v}, T\right)+\frac{1}{2}\right] .
$$

Usually the coupling coefficient $\partial E_{g} / \partial n_{v}$ is defined as $l_{v}^{2} \partial^{2} E_{g} / \partial x_{v}^{2}$ with $l_{v}$ being the characteristic length of the normal mode [35], but with this definition Eq. (4) gives a too large gap renormalization of $0.95 \mathrm{eV}$ at $500 \mathrm{~K}$, demonstrating the failure of a harmonic expansion of $E_{g}$ with respect to $x_{v}$. However Fig. 3 reveals a correlation between the calculated gap and $D_{\text {SnI }}$, the average Sn-I bond length, which accounts for the gap increase both from the electron-phonon interaction and between the unperturbed $\alpha, \beta$, and $\gamma$ phases (orange stars). Following Ref. [13] we attribute this sensitivity to a weakened Sn- $s / \mathrm{I}-p$ antibonding interaction as the bond length increases, narrowing the valence band and widening the band gap. This correlation motivates a resummation and new coupling constant definition:

$$
\frac{\partial E_{g}}{\partial n_{v}}=l_{v}^{2} \frac{d E_{g}}{d D_{\mathrm{SnI}}} \frac{\partial^{2} D_{\mathrm{SnI}}}{\partial x_{v}^{2}},
$$

where $d E_{g} / d D_{\mathrm{SnI}}$ is the gradient of the straight line in Fig. 3. In Fig. 4 we plot the spectral functions [56] $g^{2} F(\omega)=$ $\sum_{\nu} \partial E_{g} / \partial n_{\nu} \delta\left(\omega-\omega_{\nu}\right)$ for the two definitions of $\partial E_{g} / \partial n_{\nu}$, showing that (a) the harmonic expansion of $E_{g}$ predicts much larger contributions from polar modes at $6 \mathrm{meV}$, and (b) both expansions yield an important contribution to the gap renormalization from the octahedral rotations at $2.3 \mathrm{meV}$, which can only be described with an anharmonic treatment of the ground state.

Equation (5) yields a gap renormalization of $0.70 \mathrm{eV}$ for the $\mathrm{B}-\alpha$ phase at $500 \mathrm{~K}$, exactly reproducing the ensemble average of Fig. 3. Noting that the B- $\beta$ and B- $\gamma$ phases display a similar correlation of $E_{g}$ with $D_{\mathrm{SnI}}$ [35], we combined $d E_{g} / d D_{\mathrm{SnI}}$ from Fig. 3 with $\partial^{2} D_{\text {SnI }} / \partial x_{v}^{2}$ obtained from the phonon eigenvectors of these phases and found renormalizations of 


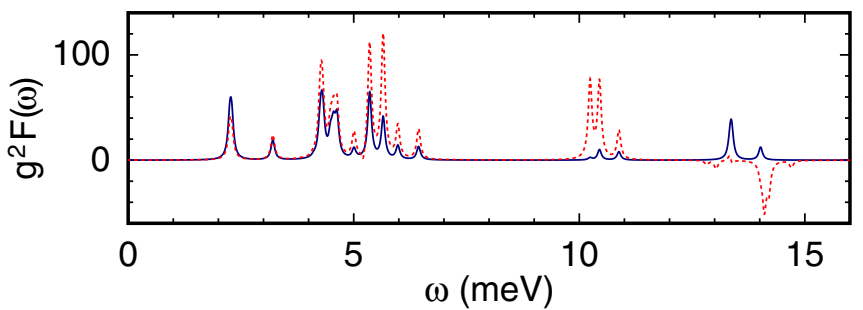

FIG. 4. (Color online) Gap spectral functions $g^{2} F(\omega)$ calculated using $\partial E_{g} / \partial n_{v}=l_{v}^{2} \partial^{2} E_{g} / \partial x_{v}^{2}$ (red dotted line) and Eq. (5) (blue solid line). The expected gap renormalization at temperature $T$ is obtained as $\int d \omega g^{2} F(\omega)\left[n_{B}(\omega, T)+1 / 2\right]$.

0.47 and $0.32 \mathrm{eV}$, also remarkably consistent with the full ensemble averages.

The surprisingly large band gap corrections raise two questions: first whether the adiabatic interpretation of $\left\langle E_{g}\right\rangle_{T}$ as the electron-phonon-corrected band gap [28] is sufficient to describe the photophysics of this polar material $[55,57]$ and second whether the supercells used to calculate the gap renormalization have introduced finite size effects (e.g., through an oversampling of the soft modes). Current methods of treating nonadiabaticity have not yet been extended to systems dominated by anharmonic couplings between different phonon modes [55,58], but we studied the finite size effect by Fourier interpolating the $\mathrm{SC} \omega$ dynamical matrix to progressively larger $N \times N \times N$ supercells of the B- $\alpha$ phase and repeating the sampling of the band gap, utilizing the localized-orbital basis sets implemented in GPAW [35,59]. We indeed observe slow convergence with supercell size, with an empirical $1 / N$ scaling. Extrapolating this behavior leads to a significant reduction of $d E_{g} / d D_{\text {SnI }}$ by $62 \%$, thus giving revised estimates of the gap renormalization from Eqs. (4) and (5) of $+0.24,+0.16$, and $+0.11 \mathrm{eV}$ for $\mathrm{CsSnI}_{3}$ at 500,380 , and $300 \mathrm{~K}$, or corrected GLLB-SC gaps of 0.90, 1.04, and $1.17 \mathrm{eV}$. Future work is required to study the nature and origin of this slow size convergence.

Connecting our work to experimental studies, we note that Ref. [8] found the peak photoluminescence (PL) to increase in energy by $0.09 \mathrm{eV}$ from 9 to $300 \mathrm{~K}$. Although this data appears to agree with our calculated shift of $+0.11 \mathrm{eV}$, we note that (a) the latter value does not include thermal expansion effects, and (b) it is unclear whether the PL corresponds to band-band transitions or defects [12,60]. At higher temperatures, our calculations indicate that the band gap will reduce, e.g., by $0.14 \mathrm{eV}$ between 380 and $500 \mathrm{~K}$. The measurement of the absorption spectrum of $\mathrm{CsSnI}_{3}$ over the 0-500 K temperature range would be highly useful to further explore these effects.

Finally, we note that while anharmonicity has been demonstrated to play a crucial role for materials at very high temperatures or pressures $[27,41]$, the conditions simulated here are relevant to the expected operating conditions for solar cells [61]. It is notable that the $0.24 \mathrm{eV}$ shift obtained for the cubic phase is of similar magnitude to the spin-orbit correction [13], with opposite sign. Our paper thus illustrates the importance of anharmonic temperature effects to the realistic modeling of the $X(\mathrm{Sn}, \mathrm{Pb}) Y_{3}$ perovskites.

We thank I. Errea, I. E. Castelli, and J. M. García-Lastra for useful discussions and acknowledge support from the Danish Council for Independent Research's Sapere Aude Program, Grant No. 11-1051390.
[1] J. D. Donaldson and J. Silver, J. Chem. Soc., Dalton Trans., 666 (1973).

[2] I. Chung, B. Lee, J. He, R. P. H. Chang, and M. G. Kanatzidis, Nature (London) 485, 486 (2012).

[3] M. M. Lee, J. Teuscher, T. Miyasaka, T. N. Murakami, and H. J. Snaith, Science 338, 643 (2012).

[4] M. H. Kumar, S. Dharani, W. L. Leong, P. P. Boix, R. R. Prabhakar, T. Baikie, C. Shi, H. Ding, R. Ramesh, M. Asta, M. Graetzel, S. G. Mhaisalkar, and N. Mathews, Adv. Mater. 26, 7122 (2014).

[5] C. C. Stoumpos, C. D. Malliakas, and M. G. Kanatzidis, Inorg. Chem. 52, 9019 (2013).

[6] Z. Chen, C. Yu, K. Shum, J. J. Wang, W. Pfenninger, N. Vockic, J. Midgley, and J. T. Kenney, J. Lumin. 132, 345 (2012).

[7] K. Shum, Z. Chen, J. Qureshi, C. Yu, J. J. Wang, W. Pfenninger, N. Vockic, J. Midgley, and J. T. Kenney, Appl. Phys. Lett. 96, 221903 (2010).

[8] C. Yu, Z. Chen, J. J. Wang, W. Pfenninger, N. Vockic, J. T. Kenney, and K. Shum, J. Appl. Phys. 110, 063526 (2011).

[9] R. D. King-Smith and D. Vanderbilt, Phys. Rev. B 49, 5828 (1994).

[10] K. Yamada, S. Funabiki, H. Horimoto, T. Matsui, T. Okuda, and S. Ichiba, Chem. Lett. 20, 801 (1991).

[11] L.-y. Huang and W. R. L. Lambrecht, Phys. Rev. B 90, 195201 (2014).
[12] I. Chung, J.-H. Song, J. Im, J. Androulakis, C. D. Malliakas, H. Li, A. J. Freeman, J. T. Kenney, and M. G. Kanatzidis, J. Am. Chem. Soc. 134, 8579 (2012).

[13] L.-y. Huang and W. R. L. Lambrecht, Phys. Rev. B 88, 165203 (2013).

[14] H. Li, I. E. Castelli, K. S. Thygesen, and K. W. Jacobsen, Phys. Rev. B 91, 045204 (2015).

[15] I. Borriello, G. Cantele, and D. Ninno, Phys. Rev. B 77, 235214 (2008).

[16] J. M. Frost, K. T. Butler, F. Brivio, C. H. Hendon, M. van Schilfgaarde, and A. Walsh, Nano Lett. 14, 2584 (2014).

[17] P. Umari, E. Mosconi, and F. De Angelis, Sci. Rep. 4, 4467 (2014).

[18] D. E. Scaife, P. F. Weller, and W. G. Fisher, J. Solid State Chem. 9, 308 (1974).

[19] J.-F. Chabot, M. Côte, and J.-F. Brière, in High Performance Computing Systems and Applications and OSCAR Symposium (Proceedings), edited by D. Sènèchal (NRC Research Press, Ottawa, 2003), p. 57.

[20] C. Yu, Y. Ren, Z. Chen, and K. Shum, J. Appl. Phys. 114, 163505 (2013).

[21] E. L. da Silva, J. M. Skelton, S. C. Parker, and A. Walsh, Phys. Rev. B 91, 144107 (2015).

[22] S. Baroni, S. de Gironcoli, A. Dal Corso, and P. Giannozzi, Rev. Mod. Phys. 73, 515 (2001). 
[23] Y. Wang, S. Shang, Z.-K. Liu, and L.-Q. Chen, Phys. Rev. B 85, 224303 (2012).

[24] D. Hooton, Philos. Mag. 46, 422 (1955).

[25] N. S. Gillis, N. R. Werthamer, and T. R. Koehler, Phys. Rev. 165, 951 (1968).

[26] S. E. Brown, I. Georgescu, and V. A. Mandelshtam, J. Chem. Phys. 138, 044317 (2013).

[27] I. Errea, M. Calandra, and F. Mauri, Phys. Rev. B 89, 064302 (2014).

[28] P. B. Allen and V. Heine, J. Phys. C: Solid State Phys. 9, 2305 (1976).

[29] J. P. Perdew, A. Ruzsinszky, G. I. Csonka, O. A. Vydrov, G. E. Scuseria, L. A. Constantin, X. Zhou, and K. Burke, Phys. Rev. Lett. 100, 136406 (2008)

[30] We used plane-wave cutoffs of 1000 and $600 \mathrm{eV}$ for the phonon and band gap calculations. We used supercells and ( $\Gamma$-centered) $k$-point grids of $2 \times 2 \times 2 / 4 \times 4 \times 4(\mathrm{~B}-\alpha), 1 \times 1 \times 2 / 6 \times 6 \times$ $4(\mathrm{~B}-\beta)$, and $1 \times 1 \times 1 / 6 \times 4 \times 6(\mathrm{~B}-\gamma)$.

[31] P. E. Blöchl, Phys. Rev. B 50, 17953 (1994).

[32] J. Enkovaara, C. Rostgaard, J. J. Mortensen, J. Chen, M. Dulak, L. Ferrighi, J. Gavnholt, C. Glinsvad, V. Haikola, H. A. Hansen, H. H. Kristoffersen, M. Kuisma, A. H. Larsen, L. Lehtovaara, M. Ljungberg, O. Lopez-Acevedo, P. G. Moses, J. Ojanen, T. Olsen, V. Petzold, N. A. Romero, J. Stausholm-Møller, M. Strange, G. A. Tritsaris, M. Vanin, M. Walter, B. Hammer, H. Häkkinen, G. K. H. Madsen, R. M. Nieminen, J. K. Nørskov, M. Puska, T. T. Rantala, J. Schiøtz, K. S. Thygesen, and K. W. Jacobsen, J. Phys.: Condens. Matter 22, 253202 (2010).

[33] G. J. Ackland, M. C. Warren, and S. J. Clark, J. Phys.: Condens. Matter 9, 7861 (1997).

[34] A. A. Maradudin and S. H. Vosko, Rev. Mod. Phys. 40, 1 (1968).

[35] See Supplemental Material at http://link.aps.org/supplemental/ 10.1103/PhysRevB.92.201205 for more details on the SC $\omega$ method and additional figures .

[36] W. Zhong, D. Vanderbilt, and K. M. Rabe, Phys. Rev. Lett. 73, 1861 (1994).

[37] J. C. Thomas and A. V. d. Ven, Phys. Rev. B 88, 214111 (2013).

[38] B. Monserrat, N. D. Drummond, and R. J. Needs, Phys. Rev. B 87, 144302 (2013).

[39] O. Hellman, I. A. Abrikosov, and S. I. Simak, Phys. Rev. B 84, 180301 (2011).

[40] D.-B. Zhang, T. Sun, and R. M. Wentzcovitch, Phys. Rev. Lett. 112, 058501 (2014)
[41] P. Souvatzis, O. Eriksson, M. I. Katsnelson, and S. P. Rudin, Phys. Rev. Lett. 100, 095901 (2008).

[42] G. N. Watson, J. Lond. Math. Soc. s1-8, 194 (1933).

[43] C. E. Patrick and F. Giustino, J. Phys.: Condens. Matter 26, 365503 (2014).

[44] As an example, a $1 \times 1 \times 1$ sampling for the B- $\gamma$ phase would require minimizing $\mathcal{F}$ with respect to 861 basis functions, compared to the 25 realized in Ref. [27].

[45] C. E. Patrick and F. Giustino, Nat. Commun. 4, 3006 (2013).

[46] S. Rossano, F. Mauri, C. J. Pickard, and I. Farnan, J. Phys. Chem. B 109, 7245 (2005).

[47] G. Antonius, S. Poncé, P. Boulanger, M. Côté, and X. Gonze, Phys. Rev. Lett. 112, 215501 (2014).

[48] C. Faber, P. Boulanger, C. Attaccalite, E. Cannuccia, I. Duchemin, T. Deutsch, and X. Blase, Phys. Rev. B 91, 155109 (2015).

[49] M. Kuisma, J. Ojanen, J. Enkovaara, and T. T. Rantala, Phys. Rev. B 82, 115106 (2010).

[50] I. E. Castelli, T. Olsen, S. Datta, D. D. Landis, S. Dahl, K. S. Thygesen, and K. W. Jacobsen, Energy Environ. Sci. 5, 5814 (2012).

[51] M. Cardona and M. L. W. Thewalt, Rev. Mod. Phys. 77, 1173 (2005).

[52] J. Serrano, C. Schweitzer, C. T. Lin, K. Reimann, M. Cardona, and D. Fröhlich, Phys. Rev. B 65, 125110 (2002).

[53] F. Giustino, S. G. Louie, and M. L. Cohen, Phys. Rev. Lett. 105, 265501 (2010).

[54] E. Cannuccia and A. Marini, Phys. Rev. Lett. 107, 255501 (2011).

[55] S. Poncé, Y. Gillet, J. Laflamme Janssen, A. Marini, M Verstraete, and X. Gonze, J. Chem. Phys. 143, 102813 (2015).

[56] R. B. Capaz, C. D. Spataru, P. Tangney, M. L. Cohen, and S. G. Louie, Phys. Rev. Lett. 94, 036801 (2005).

[57] Excluding the polar modes from the band gap sampling at $500 \mathrm{~K}$ reduced the correction by $0.09 \mathrm{eV}$ at $500 \mathrm{~K}$.

[58] G. Antonius, S. Poncé, E. Lantagne-Hurtubise, G. Auclair, X. Gonze, and M. Côté, Phys. Rev. B 92, 085137 (2015).

[59] A. H. Larsen, M. Vanin, J. J. Mortensen, K. S. Thygesen, and K. W. Jacobsen, Phys. Rev. B 80, 195112 (2009).

[60] P. Xu, S. Chen, H.-J. Xiang, X.-G. Gong, and S.-H. Wei, Chem. Mater. 26, 6068 (2014).

[61] A. Jones and C. Underwood, Solar Energy 70, 349 (2001). 